\title{
HI Imaging the Low Red-shift Cosmic Web
}

\author{
Robert Braun ${ }^{\mathrm{a}}$ \\ ${ }^{\mathrm{a} A S T R O N}$, \\ P.O. Box 2, 7990 AA Dwingeloo, The Netherlands
}

\begin{abstract}
Only in recent years has the realization emerged that galaxies do not dominate the universal baryon budget but are merely the brightest pearls of an underlying Cosmic Web. Although the gas in these inter-galactic filaments is moderately to highly ionized, QSO absorption lines have shown that the surface area increases dramatically in going down to lower HI column densities. The first image of the Cosmic Web in HI emission has just been made of the Local Group filament connecting M31 and M33. The corresponding HI distribution function is in very good agreement with that of the QSO absorption lines, confirming the 30-fold increase in surface area expected between $10^{19} \mathrm{~cm}^{-2}$ and $10^{17} \mathrm{~cm}^{-2}$. The critical observational challenge is crossing the "HI desert", the range of $\log \left(\mathrm{N}_{H I}\right)$ from about 19.5 down to 18 , over which photo-ionization by the intergalactic radiation field produces an exponential decline in the neutral fraction from essentially unity down to a few percent. Nature is kinder again to the HI observer below $\log \left(\mathrm{N}_{H I}\right)=18$, where the neutral fraction decreases only very slowly with $\log \left(\mathrm{N}_{H I}\right)$. With the SKA we can begin the systematic study of the Cosmic Web beyond the Local Group. With moderate integration times, the necessary resolution and sensitivity can be achieved out to distances beyond the Virgo cluster. When combined with targeted optical and UV absorption line observations, the total baryonic masses and enrichment histories of the Cosmic Web will be determined over the complete range of environmental over-densities.
\end{abstract}

\section{Introduction}

Extragalactic astronomy has traditionally focused on the regions of extreme cosmic overdensity that we know as galaxies. Only in recent years has the realization emerged that galaxies do not dominate the universal baryon budget but are merely the brightest pearls of an underlying Cosmic Web. Filamentary components extending between the massive galaxies are a conspicuous prediction of high resolution numerical models of structure formation (eg. Davé et al. [5], 6]). Such calculations suggest that in the current epoch, cosmic baryons are almost equally distributed by mass amongst three components: (1) galactic concentrations, (2) a warm-hot intergalactic medium (WHIM) and (3) a diffuse intergalactic medium. These three components are each coupled to a decreasing range of baryonic over-density: $\log \left(\rho_{H} / \bar{\rho}_{H}\right)>3.5,1-3.5$, and $<1$ and are probed by QSO absorption lines with specific ranges of neutral column density: $\log \left(N_{H I}\right)>18,14-18$, and $<14$. The neutral fraction is thought to decrease with decreasing column density from about $1 \%$ at $\log \left(\mathrm{N}_{H I}\right)=17$, to less than $0.1 \%$ at $\log \left(\mathrm{N}_{H I}\right)=13$. Although a very wide range of physical conditions can be found within galaxies, the WHIM is thought to be a condensed shock-heated phase with temperature in the range $10^{5}-10^{7} \mathrm{~K}$, while the diffuse IGM is predominantly photo-ionized with temperature near $10^{4} \mathrm{~K}$.

The strongest observational constraints on this picture come from the statistics of the QSO absorption lines. Enough of such QSO spectra have been obtained to allow good statistical determinations to be made of the rate of occurrence of intervening absorbers as function of their column density. By binning such data in red-shift intervals, it has even been possible to gauge the cosmic evolution of intervening absorbers ( $[\underline{8}])$. Inter-galactic space has apparently become continuously tidier by about an order of magnitude from red-shifts of several down to zero; with a decreasing cross-section of high column absorbers. At the current epoch we can now confidently pre- 
dict that in going down from $\mathrm{HI}$ column densities of $10^{19} \mathrm{~cm}^{-2}$ (which define the current "edges" of well-studied nearby galaxies in HI emission) to $10^{17} \mathrm{~cm}^{-2}$, the surface area will increase by a factor of 30 . The critical observational challenge is crossing the "HI desert", the range of $\log \left(\mathrm{N}_{H I}\right)$ from about 19.5 down to 18 , over which photoionization by the intergalactic radiation field produces an exponential decline in the neutral fraction from essentially unity down to a few percent (eg. Dove \& Shull [7]). Nature is kinder again to the HI observer below $\log \left(\mathrm{N}_{H I}\right)=18$, where the neutral fraction decreases only very slowly with $\log \left(\mathrm{N}_{H I}\right)$. The baryonic mass traced by this gas (with a $1 \%$ or less neutral fraction) is expected to be comparable to that within the galaxies, as noted above.

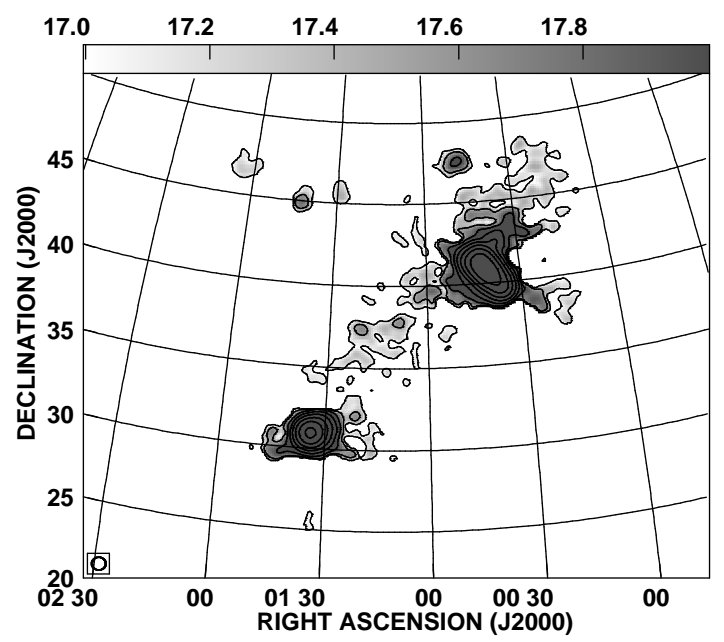

Figure 1. Integrated HI emission from features which are kinematically associated with M31 and M33. The grey-scale varies between $\log \left(\mathrm{N}_{H I}\right)=17-18$, for $\mathrm{N}_{H I}$ in units of $\mathrm{cm}^{-2}$. Contours are drawn at $\log \left(\mathrm{N}_{H I}\right)=17,17.5,18, \ldots 20.5 . \quad$ M31 is located at $(\mathrm{RA}, \mathrm{Dec})=\left(00: 43,+41^{\circ}\right)$ and M33 at $(\mathrm{RA}, \mathrm{Dec})=\left(01: 34,+30^{\circ}\right)$, The two galaxies are connected by a diffuse filament joining the systemic velocities.
But how are these diffuse systems distributed and what are their kinematics? These are questions which can not be addressed with the QSO absorption line data. The areal density of suitable background sources is far too low to allow "imaging" of the intervening low column density systems in absorption. Direct detection of the free-free continuum or recombination line emission from the ionized gas has also proven well beyond the capabilties of current X-ray and optical instrumentation. For example the expected $\mathrm{H} \alpha$ emission measure is only about $\mathrm{EM}=5 \times 10^{-4}$ $\mathrm{cm}^{-6}$ pc. The very best current $\mathrm{H} \alpha$ imaging results reach down to about $\mathrm{EM}=0.1 \mathrm{~cm}^{-6} \mathrm{pc}$, which is still orders of magnitude removed from what would be needed.

\section{The First Cosmic Web Images}

Although conventional imaging in the $21 \mathrm{~cm}$ emission line of neutral hydrogen has not typically reached column densities below about $10^{19}$ $\mathrm{cm}^{-2}$, this is not a fundamental limitation. Long integrations with an (almost-)filled aperture can achieve the required brightness sensitivity to permit direct imaging of the small neutral fraction within the Cosmic Web filaments between galaxies. The first detection of such diffuse filaments in the extended environment of M31 has just been made by Braun \& Thilker ([1]). This was accomplished by utilizing total power measurements made with the fourteen $25 \mathrm{~m}$ dishes of the Westerbork Synthesis Radio Telescope (WSRT). A series of drift-scan observations were used to obtain Nyquist-sampled HI imaging of a region $60 \times 30$ degrees region in extent, centered approximately on the position of M31. Although the angular resolution is low (effective beam of 49 arcmin, corresponding to $11 \mathrm{kpc}$ at the M31 distance) the column density sensitivity is very high $\left(4 \times 10^{16} \mathrm{~cm}^{-2} \mathrm{rms}\right.$ over $\left.17 \mathrm{~km} \mathrm{~s}^{-1}\right)$. A diffuse filament is detected connecting the systemic velocities of M31 to M33 (at a projected seperation of $200 \mathrm{kpc}$ ) and also extending away from M31 in the anti-M33 direction as shown in Figure 1. This diffuse filament appears to be fueling denser gaseous streams and filaments in the outskirts of both galaxies. Peak neutral column densities 
within the filament only amount to some $3 \times 10^{17}$ $\mathrm{cm}^{-2}$. The extremely diffuse nature of the $\mathrm{HI}$ has been confirmed by pointed Green Bank Telescope (GBT) observations of a local peak in the filament which yields the same low peak column density $\left(3 \times 10^{17} \mathrm{~cm}^{-2}\right)$, despite a telescope beam area that is 25 times smaller.

The interaction zone of the diffuse filament with M31 has been studied in complimentary surveys: a $6 \times 6$ degree field imaged with the GBT (Thilker et al 9]) and a $5 \times 2$ degree WSRT mosaic of nearly 200 synthesis pointings (Braun et al [2, 3]). Our three surveys permit calculation of the HI distribution function from HI emission measurements (rather than QSO absorption measurements) over an unprecedented range in $\log \left(\mathrm{N}_{H I}\right)=17.2$ to $\log \left(\mathrm{N}_{H I}\right)=21.9$ as shown in Figure 2. The $\mathrm{N}_{H I}$ data for the M31 environment were normalized to the average space density of galaxies using the HIMF of Zwaan et al. ([10]).

The HI distribution function of these structures agrees very well with that of the low red-shift QSO absorption lines which are also plotted in the figure as filled circles with error bars. The predicted factor of 30 increase in surface covering factor for low $\mathrm{N}_{H I}$ emission has been observationally verified. In so doing, it has been possible to provide the first image of a Lyman Limit absorption system. The morphology and kinematics are fully in keeping with the cosmic web hypothesis outlined above. We are now in a position to witness the continuing gaseous fueling of normal galaxies with direct imaging.

\section{The SKA and the Cosmic Web}

The exquisite sensitivity of the SKA will permit the direct kinematic imaging of the Cosmic Web in a broad range of galaxy environments. Tapering of the total collecting area to an angular resolution of about 150" will provide a brightness sensitivity of $8 \mathrm{mK}$ over $1 \mathrm{~km} \mathrm{~s}^{-1}$ in an hour of integration. A four hour integration will then suffice to achieve an HI column density sensitivity of $3.2 \times 10^{16} \mathrm{~cm}^{-2} \mathrm{rms}$ over the typical $20 \mathrm{~km} \mathrm{~s}^{-1}$ linewidth. The 150" beamsize corresponds to a physically interesting linear resolution of better than $15 \mathrm{kpc}$ out to distances of $21 \mathrm{Mpc}$. The ex- tended environments of thousands of galaxies; be they isolated, in loose groups or even in the Virgo cluster are all within reach.

As a specific example of the type of study that will be possible, consider a blind fully-sampled survey of a portion of the super-galactic plane filament between RA $=12.2$ and 12.9 hours and Dec. $=+2$ and $+10^{\circ}$. More than 130 galaxies with associated HI, covering the full range of Hubble types, are currently known in this region at distances lying between the Local Group and the Virgo cluster. A complete imaging survey of this region can be obtained with some 80 pointings (of a 1.1 square degree instantaneous FOV) reaching a column density depth of $3.2 \times 10^{16} \mathrm{~cm}^{-2}$ rms over $20 \mathrm{~km} \mathrm{~s}^{-1}$ in 300 hours of integration. Determination of gaseous fueling rates will likely need to be estimated statistically, since only the radial (and not the transverse) velocity components can be directly measured. Hence the need for large samples of galaxies. Once such an imaging study has been obtained, a complimentary directed study can be carried out of optical and UV absorption toward suitably located background QSO's behind individual filaments. In this way the metallicity and ionization state of the gas can be established. With this information, total baryonic masses and enrichment histories of the Cosmic Web can be determined over the complete range of environmental over-density.

\section{REFERENCES}

1. Braun, R., Thilker, D.A., 2004 A\&A, 417, 421.

2. Braun, R., Thilker, D. A., Corbelli, E., Walterbos, R.A.M., 2002, http://www.astron.nl/newsletter/2002-1/index.html

3. Braun, R., Thilker, D. A., Corbelli, E., Walterbos, R. A. M., 2004, in prep.

4. Corbelli, E., Bandiera, R., 2002, ApJ, 567, 712 .

5. Davé, R., Hernquist, L., Katz, N., Weinberg, D. H., 1999, ApJ, 511, 521.

6. Davé, R., Cen, R., Ostriker, J. P., et al., 2001, ApJ, 552, 473.

7. Dove, J. B., Shull, J. M., 1994, ApJ 423, 196.

8. Storrie-Lombardi, L. J., 2000, ApJ 543, 552. 
9. Thilker, D.A., Braun, R., Walterbos, R. A. M., et al. 2004 ApJ, 601, L39.

10. Zwaan, M. A., Staveley-Smith L., Koribalski, B. S., et al. 2003, AJ, 125, 2842.

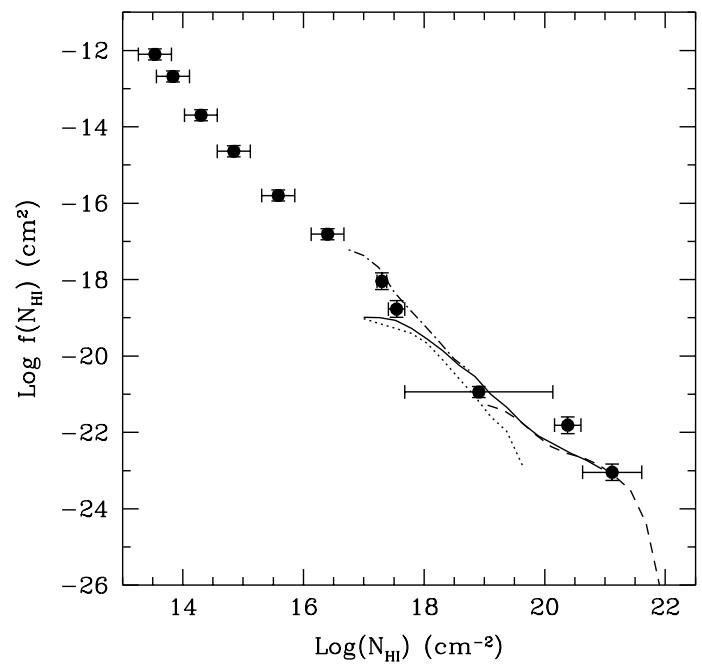

Figure 2. The distribution function of HI column density due to M31 and it's environment. The data from three HI surveys of M31 are combined in this figure to probe column densities over a total range of some five orders of magnitude. The dashed line is from the WSRT mosaic (Braun et al. 3 ) with $1^{\prime}$ resolution over $80 \times 40 \mathrm{kpc}$, the dotted and solid lines from our GBT survey (Thilker et al. 9]) with $9^{\prime}$ resolution over $95 \times 95 \mathrm{kpc}$ and the dot-dash line from the wide-field WSRT survey (Braun \& Thilker [1]) with $48^{\prime}$ resolution out to $150 \mathrm{kpc}$ radius. The filled circles with errorbars are the low red-shift QSO absorption line data as tabulated by Corbelli \& Bandiera (幽). 